\title{
Stabilizing Controller Design for a Special Class of PWA Systems using Discontinuous Piecewise Quadratic Lyapunov Functions
}

\author{
Hesam Sajjadi \\ Electrical Engineering Ms, \\ Yong Researchers and Elite \\ Club, Mashhad Branch, Islamic \\ Azad University, Mashhad, \\ Iran
}

\author{
Reyhaneh Kardehi \\ Moghaddam \\ Electrical Engineering \\ Department, Mashhad Branch, \\ Islamic Azad University, \\ Mashhad, Iran
}

\author{
Najmeh Eghbal \\ Electrical Engineering Group \\ Sadjad University of \\ Technology, Iran
}

\begin{abstract}
In this paper a new controller is proposed to stabilize an especial class of hybrid piecewise affine systems. In this study, for the first time, the stabilizing controller is designed based on discontinuous piecewise quadratic Lyapunov functions which decrease the conservation and propose a wider class of applicable Lyapunov functions as it omits the continuity condition in boundary points compared to continuous piecewise quadratic Lyapunov functions. In addition the stability conditions are formulated in the form of Bilinear Matrix Inequalities (BMI) problem. To solve the proposed problem, BMI is defined in the form of a multi-objective nonlinear optimization problem which has been solved through using genetic Algorithm (GA).
\end{abstract}

\section{Keywords}

Bilinear matrix inequalities, discontinuous piecewise quadratic lyapunov function, multiple lyapunov function, piecewise affine.

\section{INTRODUCTION}

Hybrid systems are a class of dynamical systems which consist of continuous and discontinuous dynamics which are implemented for modeling of systems. The capability of hybrid systems to model the special systems behaviors is a great point of interest. Piecewise affine systems (PWA) are a class of hybrid systems which contain affine subsystems and switching rules [1,2]. Since 1970, PWA systems have been proposed as an effective tool for design and analysis of the systems [3-5]. PWA systems are special class of hybrid systems that can be described by PWA equations [6,7]. They are highly applicable considering the fact that most of nonlinear behaviors of physical systems such as saturation, dead zone, relay, and hysteresis are modeled by PWA systems $[3,8-10]$. One advantage of describing physical systems with PWA models is that they can easily be converted to piecewise linear (PWL) systems which have a simple structure. Most of PWA systems are continuous as they are employed to describe the continuous-time nonlinear systems [11,12]. Some physical PWA systems such as dead and saturation systems are naturally continuous[13]. The special class which is considered in this paper is two dimensioned PWA systems. The proposed idea to design a controller for such systems is based on discontinuous piecewise quadratic (PWQ) Lyapunov functions. Based on the fact that continuous PWQs are contained by discontinuous PWQ Lyapunov functions, using discontinuous PWQs will decrease the conservation as the search space to find such functions is wider in comparison to continuous PWQs. In this paper the sufficient stability conditions which leads to the design of stabilizer controller are performed in the form of a Bilinear Matrix Inequality (BMI) problem. To solve such a problem, BMI is converted to a nonlinear multi objective problem which can easily be solved by using optimization methods such as GA. In the sequel in section 2 the preliminaries are defined. The Lyapunov based stabilizing techniques are proposed in section 3. The new idea of using discontinuous PWQ Lyapunov functions for designing a controller for PWA systems is defined in section 4. Simulation analysis and conclusions are proposed in sections 5 and 6 .

\section{PRELIMINARIES}

In this part, the primary definitions and theorems are proposed.

\subsection{Definition1: PWA Systems}

The state equations of a PWA system in $x \subset R^{2}$ is described as follows [14-15]:

$\dot{x}(t)=A_{i} x(t)+a_{i}+B_{i} u(t) \quad x(t) \in \bar{X}_{i}$

Where $x(t) \in X \subset R^{n}$ and $u(t) \in U \subset R^{m}$ are state variables and controlling signals respectively. $\mathrm{A}_{\mathrm{i}}$ and $\mathrm{a}_{\mathrm{i}}$ are constant Matrix/vector [2].

\subsection{Definition2: State Space Partitioning}

In $\left\{X_{i}\right\}_{i \in I}$, I includes all subspaces If $i \in I_{0}$, then $I_{0}$ is the subspace that contains the equilibrium point [2].

$i \in I_{0}, I_{0}=\left\{i \in I: 0 \in \bar{X}_{i}\right\}$

Where the subspaces can be described as [2]: $\left.\bar{X}_{i}=\left\{x \in R^{2}: E_{i} x+e_{i}\right\rangle 0\right\} \quad i \in I \quad$ (3) Where

$E_{i}$ and $e_{i}$ are constant matrix and vectors respectively.

\subsection{Definition3: Boundary Points of Two} Subspaces

$x^{*}$ is aboundary point between $X_{i}$ and $X_{j}$ subspaces, if $\bar{X}_{i} \cap \bar{X}_{j} \neq 0$ equivalently:

$x^{*} \in \bar{X}_{i} \cap \bar{X}_{j} \subseteq\left\{x \mid x=F_{i j} s+f_{i j}, s \in R\right\}$

Lemma 1: The PWA systems of (1) can be represented by a PWL system . 


$$
\begin{aligned}
& \text { Proof: } \bar{x}=\left[\begin{array}{ll}
x^{T} & 1
\end{array}\right]^{T}, \bar{A}_{i}=\left[\begin{array}{cc}
A_{i} & a_{i} \\
0 & 0
\end{array}\right], \bar{E}_{i}=\left[\begin{array}{ll}
E_{i} & e_{i}
\end{array}\right] \\
& \bar{F}_{i j}=\left[\begin{array}{cc}
F_{i j} & f_{i j} \\
0 & 1
\end{array}\right], \bar{B}_{i}=\left[\begin{array}{c}
B_{i} \\
0
\end{array}\right]
\end{aligned}
$$

The system (1) can be rewritten as:

$$
\dot{\bar{x}}=\bar{A}_{i} \bar{x}+\bar{B}_{i} \bar{u} \quad x \in \bar{X}_{i}
$$

It is clear that in (4) if $F_{i j} \neq 0$ the boundary is a line and if $F_{i j}=0$ the boundary is in the form of a single node. Considering $\bar{X}_{i} \cap \bar{X}_{j} \neq 0$ and $F_{i j} \neq 0, C_{i j}$ and $S_{i j}$ are defined as $\bar{C}_{i j}=\left[\begin{array}{ll}C_{i j} & c_{i j}\end{array}\right], S_{i j}=\left\{x \mid \bar{C}_{i j} \bar{x}=0\right\}$ where $C_{i j}$ is a normal vector in $S_{i j}$ form $X_{i}$ to $X_{j}$.

\subsection{Definition 4: Continuity of PWA systems}

The described system (1) is continuous if the direction of the state vectors does not change in the boundary between the two regions.

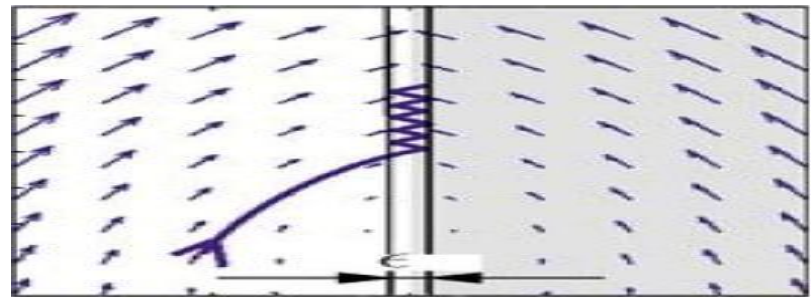

Fig.1. Sliding mode in the boundary between the two regions

\subsection{Definition 5: Convex Set}

The set $C$ is convex if every line which connects two arbitrary points be completely contained by $\mathrm{C}$. in other words the following relation should be satisfied [16]:

$$
\begin{aligned}
& \theta_{1} x_{1}+\theta_{2} x_{2}+\ldots .+\theta_{k} x_{k} \in C \\
& \sum_{i=1}^{k} \theta_{i}=1, \theta_{i} \geq 0
\end{aligned}
$$

The analysis and design problems of PWA systems can be formulated in the form of a convex problem or linear matrix inequalities [3] with the form below:

$$
F(x)=F_{0}+\sum_{i=1}^{m} x_{i} F_{i}>0
$$

Where $x=\left[x_{1}, \ldots, x_{m}\right] \in R^{m}$ is the vector of unknown parameters and $F_{i}=F_{i}^{T} \in R^{n \times n}$ is defined symmetric matrix.

\section{DESIGNING THE STABILITY CONTROLLER}

In this part the controller is designed based on discontinuous PWQ Lyapunov functions so as to asymptotically stabilize the close loop system.
It is considered that system (1) has a controller with the following structure:

$u=k_{i} x+m_{i}=\bar{K}_{i} \bar{x}$

Where,

$$
\bar{K}_{i}=\left[\begin{array}{ll}
k_{i} & m_{i}
\end{array}\right]
$$

Substituting (8) and (9) in (1) the close loop system has the following form:

$$
\dot{\bar{x}}=\left(\bar{A}_{i}+\bar{B}_{i} \bar{k}_{i}\right) \bar{x}
$$

After indicating the controller structure, the stability controller will be designed using Lyapunov stability theorems. It is clear that existence of a Lyapunov function is the sufficient stability condition, thus the disability to find a proper Lyapunov function doesn't lead to instability of the system.

Theorem 1. Lyapunov direct stability theorem

Consider system $\dot{x}=f(x, u)$ with a point of equilibrium at x=0.Consider a function $V(x): R^{n} \rightarrow R$ such that satisfies the following condition:

$$
V(\bar{x})=0 \text { for } x=0
$$

Then the equilibrium is asymptotically stable.

One of the most famous Lyapunov functions is the common Quadratic (CQ) one [17 - 21]. In the next part the stability analysis based in CQ functions is described.

\subsection{CQ Lyapunov based Stability Analysis} In this method the CQ Lyapunov function is considered as:

$$
V(x)=x^{T} p x
$$

According to theorem 1 the sufficient stability conditions are as follows:

$$
\begin{aligned}
& \text { a) } V(x)>0 \rightarrow P>0 \\
& \text { b) } \dot{V}(x)<0 \rightarrow\left(\bar{A}_{i}+\bar{B}_{i} \bar{K}_{i}\right)^{T} P+P\left(\bar{A}_{i}+\bar{B}_{i} \bar{K}_{i}\right)<0 \forall i \in I
\end{aligned}
$$

Where the $\mathrm{b}$ condition in (13) is usually described as:

$$
\begin{aligned}
& \left(\bar{A}_{i}+\bar{B}_{i} \bar{K}_{i}\right)^{T} P+P\left(\bar{A}_{i}+\bar{B}_{i} \bar{K}_{i}\right)=-Q \\
& P=P^{T}>0 \\
& Q=Q^{T}>0
\end{aligned}
$$

It should be considered that CQ Lyapunov functions are highly conservative and in some cases it is not possible to find a Lyapunov function for special subspaces. According to mentioned limitations we propose the Piecewise Quadratic (PWQ) Lyapunov functions. Using this functions, one can define a special Lyapunov function for each subspace which reduces the conservatism. These functions have the following structure:

$$
V(x)=V_{i}(x)=\bar{x}^{T} \bar{p}_{i} \bar{x} \quad \forall x \in \bar{X}_{i}
$$

It is clear that for such structure the stability of all subsystems is just a necessary stability condition and the special switching conditions which are mentioned in [10,13,22 - 23] should also be satisfied. The most common classes of PWQs are continuous 
piecewise Quadratic (CPWQ) and discontinuous PWQs (DPWQ) .Where CPWQs are usually denoted by PWQs in literatures.

\subsection{PWQ Lyapunov based Stability Analysis}

As mentioned in the previous section, using PWQs decreases the conservatism comparedto CQs, as the PWQs should be specified for each subsystem individually. Despite the mentioned benefits, CPWQs have some limitations in stability analysis. Although Lyapunov function is determined for each subspace, it should be continuous in the boundary between the two regions and this fact shrinks the class of nonlinear systems which can be analyzed by CPWQs The continuity of PWQs in boundary regions is defined in the form of proposition1.

Proposition 1: The necessary and sufficient continuity condition of PWQs at boundary node is [24,25] :

$V_{i}(x)=V_{j}(x) \rightarrow \bar{x}^{T} \bar{p}_{i} \bar{x}=\bar{x}^{T} \bar{p}_{j} \bar{x}$

Substituting $\bar{x}=\bar{F}_{i j} \bar{s}$ in (17) yields:

$V_{i}(x)=V_{j}(x) \rightarrow \bar{s}^{T} \bar{F}_{i j}{ }^{T} \bar{p}_{i} \bar{F}_{i j} \bar{s}=\bar{s}^{T} \bar{F}_{i j}{ }^{T} \bar{p}_{j} \bar{F}_{i j} \bar{s}$

Proposition 2: After satisfyingthe continuity condition, the sufficient stability condition of close loop system (10) based on CPWQs Lyapunov function is [13]:

$$
\left\{\begin{array}{c}
I_{n}\left(\left(\bar{A}_{i}+\bar{B}_{i} \bar{K}_{i}\right)^{T} \bar{P}_{i}+\bar{P}_{i}\left(\bar{A}_{i}+\bar{B}_{i} \bar{K}_{i}\right)+\bar{E}_{i}^{T} \bar{u}_{i} \bar{E}_{i}\right) I_{n}\langle 0 \\
\left.I_{n}\left(\bar{P}_{i}-\bar{E}_{i}^{T} \bar{w}_{i} \bar{E}_{i}\right) I_{n}\right\rangle 0 \\
\bar{A}_{i}=\left[\begin{array}{cc}
A_{i} & 0 \\
0 & 0
\end{array}\right], \bar{P}_{i}=\left[\begin{array}{cc}
P_{i} & 0 \\
0 & 0
\end{array}\right], I_{n}=\left[\begin{array}{ll}
1 & 0 \\
0 & 1
\end{array}\right]
\end{array}\right.
$$

$$
\left\{\begin{array}{c}
\left(\bar{A}_{i}+\bar{B}_{i} \bar{K}_{i}\right) \bar{P}_{i}+\bar{P}_{i}\left(\bar{A}_{i}+\bar{B}_{i} \bar{K}_{i}\right)+\bar{E}_{i}^{T} \bar{u}_{i} \bar{E}_{i}<0 \\
\bar{P}_{i}-\bar{E}_{i}^{T} \bar{w}_{i} \bar{E}_{i}>0 \\
\bar{A}_{i}=\left[\begin{array}{cc}
A_{i} & a_{i} \\
0 & 0
\end{array}\right], \bar{P}_{i}=\left[\begin{array}{cc}
P_{i} & q_{i} \\
q_{i}^{T} & r_{i}
\end{array}\right]
\end{array}\right.
$$$$
I_{0}=\left\{i \in I: 0 \in \bar{X}_{i}\right\}
$$$$
I_{1}=\left\{i \in I: 0 \notin \bar{X}_{i}\right\}
$$

Proposition3: The following equality leads to continuity of CPWQs on boundary nods [26-27]. $\left(\bar{A}_{i}+\bar{B}_{i} \bar{K}_{i}\right) \bar{F}_{i j}=\left(\bar{A}_{j}+\bar{B}_{j} \bar{K}_{j}\right) \bar{F}_{i j}$

According to the above propositions, we come to the conclusion that omitting the continuity condition of CPWQs can effectively to expand the class of discussed nonlinear systems and this is possible by replacing CPWQs by DPWQs. In this paper, for the first time, we apply the DPWQs for designing controller and stability analysis of the close loop system (10). In the next chapter we will show that using this structure sufficiently decreases the conservatism and expands the field of study.

\section{DPWQS BASED STABILITY ANALYSIS}

According to $[22,28]$, if the PWQ function decreases in switching times, the system will be asymptotically stable [2].
For a hybrid system (22), the switching happens when the state trajectory goes across the boundary where (4) is defined as the switching surface.

$$
\dot{x}=f_{i}(x) \quad x \in \bar{X}_{i}, i \in I
$$

Theorem2: The sufficient stability condition of a continuous system (22) based on discontinuous PWQs is presented:

$$
\begin{aligned}
& f_{i}(x)=f_{j}(x) \quad x \in \bar{X}_{i} \cap \bar{X}_{j} \\
& \left\{\begin{array}{l}
\text { a) } V_{i}(x)=0 \quad x=0, i \in I_{0} \\
\text { b) } V_{i}(x)>0 \quad x \in \bar{X}_{i}, x \neq 0, i \in I
\end{array}\right.
\end{aligned}
$$

$\dot{V}_{i}(x)<0 \quad x \in \bar{X}_{i}, x \neq 0, i \in I$

$V_{i}(x)-V_{j}(x)=w_{i j}{ }^{1} C_{i j} f_{i}(x)+w_{i j}{ }^{2} C_{i j} f_{j}(x) \quad F_{i j} \neq 0$,

$\forall i \in I, j \in N_{i}, x \in \bar{X}_{i} \cap \bar{X}_{j}$

$N_{i}=\left\{K \in I, K \neq i, \bar{X}_{i} \cap \bar{X}_{k} \neq 0\right\}$

It has asymptotic stability if a selection of functions $V_{i}(x): \bar{x}_{i} \rightarrow R, i \in I$ and nonnegative scalars, $w_{i j}{ }^{1}, w_{i j}{ }^{2}$, exist which satisfy (24)-(26) [2].

\subsection{Controller design based on DPWQS}

In this paper, the new approach of using DPWQS for stability analysis and controller design is proposed [2].

As this method ignores the continuity condition in boundary nodes, it expands the search space of Lyapunov functions and decreases the conservatism effectively.

The structure of DPWQs is previously defined in (15) where $\bar{P}_{i}=\bar{P}_{i}^{T} \in R^{3 \times 3}$ :

$\bar{P}_{i}=\left[\begin{array}{cc}p_{i} & q_{i} \\ q_{i}^{T} & r_{i}\end{array}\right] \quad \forall i \in I$

where $p_{i} \in R^{2 \times 2}, q_{i} \in R^{2}, r_{i} \in R$.

The sufficient and necessary stability conditions based on DPWQs is formulated by theorem 3 .

Theorem3: Let $\bar{W}_{i}, \bar{U}_{i}, i \in I$ be unknown matrices with nonnegative inputs and $\bar{w}_{i j}{ }^{k}, k=1, \ldots, 2, i, j \in I$ be unknown vectors with negative inputs. Consider the following definitions:

$$
\begin{aligned}
& \bar{H}_{i j}=\bar{E}_{i}^{T} \bar{w}_{i j}{ }^{1} \bar{C}_{i j}\left(\bar{A}_{i}+\bar{B}_{i} \bar{K}_{i}\right)+\bar{E}_{j}{ }^{T} \bar{w}_{i j}{ }^{2} \bar{C}_{i j}\left(\bar{A}_{j}+\bar{B}_{j} \bar{K}_{j}\right) \\
& \bar{L}_{i}=\bar{E}_{i}^{T} \bar{U}_{i} \bar{E}_{i} \\
& \bar{M}_{i}=\bar{E}_{i}^{T} \bar{W}_{i} \bar{E}_{i}
\end{aligned}
$$

If there exists a choice for $\bar{W}_{i}, \bar{U}_{i}, \bar{P}_{i}$ and $\bar{w}_{i j}{ }^{k}, k=1, \ldots, 2, i, j \in I$ that satisfies conditions (29) to (34) then the described system (10) will be asymptotically 
stable and converges to equilibrium point and the proposed controller will be a stabilizer.

$$
\begin{aligned}
& \bar{p}_{i}=\left[\begin{array}{cc}
p_{i} & q_{i} \\
q_{i}{ }^{T} & r_{i}
\end{array}\right] \forall i \in I, \bar{p}_{i}=\left[\begin{array}{cc}
p_{i} & 0 \\
0 & 0
\end{array}\right] \forall i \in I_{0} \\
& \bar{P}_{i}-\bar{L}_{i}>0 \quad \forall i \in I, i \notin I_{0} \\
& {\left[\begin{array}{ll}
I_{n} & 0
\end{array}\right]\left(\bar{p}_{i}-\bar{L}_{i}\right)\left[\begin{array}{c}
I_{n} \\
0
\end{array}\right]>0 \quad \forall i \in I_{0}} \\
& \left(\bar{A}_{i}+\bar{B}_{i} \bar{K}_{i}\right)^{T} \bar{p}_{i}+\bar{p}_{i}\left(\bar{A}_{i}+\bar{B}_{i} \bar{K}_{i}\right)+\bar{E}_{i}{ }^{T} \bar{W}_{i} \bar{E}_{i}<0 \\
& \forall i \in I, i \notin I_{0}
\end{aligned}
$$

$\left[\begin{array}{ll}I_{n} & 0\end{array}\right]\left(\left(\bar{A}_{i}+\bar{B}_{i} \bar{K}_{i}\right)^{T} \bar{p}_{i}+\bar{p}_{i}\left(\bar{A}_{i}+\bar{B}_{i} \bar{K}_{i}\right)+\bar{M}_{i}\right)\left[\begin{array}{c}I_{n} \\ 0\end{array}\right]<0$

$\forall i \in I_{\mathrm{O}}$

$$
\left(\bar{A}_{i}+\bar{B}_{i} \bar{K}_{i}\right) \bar{F}_{i j}=\left(\bar{A}_{j}+\bar{B}_{j} \bar{K}_{j}\right) \bar{F}_{i j}
$$

It is clear that the DPWQs should be decreased in boundary nodes. Thus, the following conditions should be satisfied:

$\bar{F}_{i j}^{T}\left(\bar{p}_{i}-\bar{p}_{j}\right) \bar{F}_{i j}=\bar{F}_{i j}{ }^{T}\left(\bar{H}_{i j}+\bar{H}_{i j}{ }^{T}\right) \bar{F}_{i j} \quad \forall i \in I, j \in N_{i}$

Where:

$$
F_{i j} \neq 0, N_{i}=\left\{K \in I, K \neq i, \bar{X}_{i} \cap \bar{X}_{k} \neq 0\right\}
$$

Proof: Related to (15) condition (29) is equal to (24-a). Where $\bar{x}^{T} \bar{L}_{i} \bar{x}>0, \forall x \in \bar{X}_{i}, i \in I$ and (30) and (31) are equal to (24-b).

Where $\left.\overline{\boldsymbol{x}}^{T} \overline{\boldsymbol{M}}_{i} \overline{\boldsymbol{x}}\right\rangle \mathrm{O}, \forall x \in \bar{X}_{i}, i \in \boldsymbol{I}$, (32) and (33) is equal to (25) and (34) is equal to (23).

$$
\begin{aligned}
& \bar{s}^{T} \bar{F}_{i j}^{T}\left(\bar{P}_{i}-\bar{P}_{j}\right) \bar{F}_{i j} \bar{s}=\bar{s}^{T} \bar{F}_{i j}^{T}\left(\bar{H}_{i j}+\bar{H}_{i j}{ }^{T}\right) \bar{F}_{i j} \bar{s} \\
& \forall i \in I, j \in N_{i}, F_{i j} \neq \mathrm{O}
\end{aligned}
$$

Therefore $\forall x \in \bar{X}_{i} \cap \bar{X}_{j}$ :

$\bar{x}^{T}\left(\bar{P}_{i}-\bar{P}_{j}\right) \bar{x}=\bar{x}^{T}\left(\bar{H}_{i j}+\bar{H}_{i j}{ }^{T}\right) \bar{x}$

Or,

$$
\begin{aligned}
& V_{i}(x)-V_{j}(x)=\bar{x}^{T} \bar{E}_{i}{ }^{T} \bar{w}_{i j}{ }^{1} \bar{C}_{i j}\left(\bar{A}_{i}+\bar{B}_{i} \bar{K}_{i}\right) \bar{x}+ \\
& \bar{x}^{T} \bar{E}_{j}{ }^{T} \bar{w}_{i j}{ }^{2} \bar{C}_{i j}\left(\bar{A}_{j}+\bar{B}_{j} \bar{K}_{j}\right) \bar{x}+ \\
& \bar{x}^{T}\left(\bar{A}_{i}+\bar{B}_{i} \bar{K}_{i}\right)^{T} \bar{C}_{i j}{ }^{T}\left(\bar{w}_{i j}{ }^{1}\right)^{T} \bar{E}_{i} \bar{x}+ \\
& \bar{x}^{T}\left(\bar{A}_{i}+\bar{B}_{i} \bar{K}_{i}\right)^{T} \bar{C}_{i j}{ }^{T}\left(\bar{w}_{i j}{ }^{2}\right)^{T} \bar{E}_{j} \bar{x} \\
& x \in \bar{X}_{i} \cap \bar{X}_{j}
\end{aligned}
$$

For $x \in \bar{X}_{i} \cap \bar{X}_{j}$ we have $\bar{E}_{i} \bar{x} \geq 0$ and $\bar{E}_{j} \bar{x} \geq 0$, so $\left(\bar{w}_{i j}{ }^{1}\right)^{T} \bar{E}_{i} \bar{x}$ and $\left(\bar{w}_{i j}{ }^{2}\right)^{T} \bar{E}_{j} \bar{x}$ are nonnegative scalars [2].

On the other hand,

$$
\begin{aligned}
& \bar{C}_{i j}\left(\bar{A}_{i}+\bar{B}_{i} \bar{K}_{i}\right) \bar{x}=\left[\begin{array}{ll}
C_{i j} & c_{i j}
\end{array}\right]\left(\left[\begin{array}{cc}
A_{i} & a_{i} \\
0 & 0
\end{array}\right]+\left[\begin{array}{c}
B_{i} \\
0
\end{array}\right]\left[\begin{array}{ll}
k_{i} & m_{i}
\end{array}\right]\right)\left[\begin{array}{c}
x \\
1
\end{array}\right]= \\
& C_{i j}\left(\left(A_{i} x+a_{i}\right)+B_{i}\left(k_{i}(x)+m_{i}\right)\right)
\end{aligned}
$$

So (37) is equal to (26) which leads to asymptotic stability of (10).

\section{SIMULATION}

In this paper the considered PWA system is a two dimensional saturation system where the he saturation constructed the nonlinear part :

$$
\begin{aligned}
& \dot{x}_{1}=-4 x_{1}+4 x_{2}-\operatorname{sat}\left(-3 x_{1}+5 x_{2}\right) \\
& \dot{x}_{2}=2 x_{1}-6 x_{2}+\operatorname{sat}\left(-3 x_{1}+5 x_{2}\right)
\end{aligned}
$$

The saturation has the following form:

$$
\begin{aligned}
& \text { 1) }-3 x_{1}+5 x_{2}>1 \rightarrow \operatorname{sat}\left(-3 x_{1}+5 x_{2}\right)=1 \\
& \text { 2) }-1<-3 x_{1}+5 x_{2}<1 \rightarrow \operatorname{sat}\left(-3 x_{1}+5 x_{2}\right)=-3 x_{1}+5 x_{2} \\
& \text { 3) }-3 x_{1}+5 x_{2}<-1 \rightarrow \operatorname{sat}\left(-3 x_{1}+5 x_{2}\right)=-1
\end{aligned}
$$

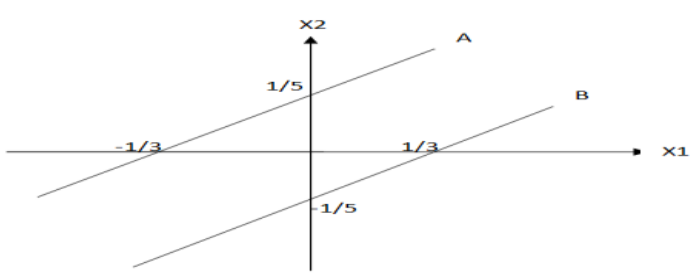

\section{Fig.2.State space partitions}

In Figure 2 , the system (39) is divided to 3 subspaces. The stability analysis is done based on DPWQs. Figures 3 to 5 show that the system is unstable in the absence of controller.

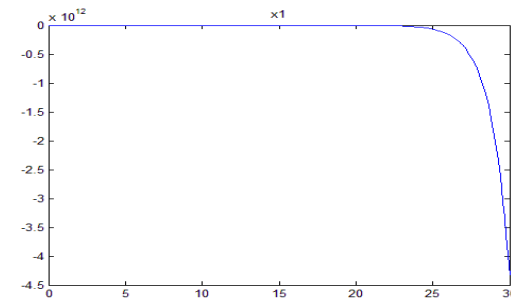

Fig.3. variations of ${ }^{x_{1}}$ related to $\mathrm{t}$

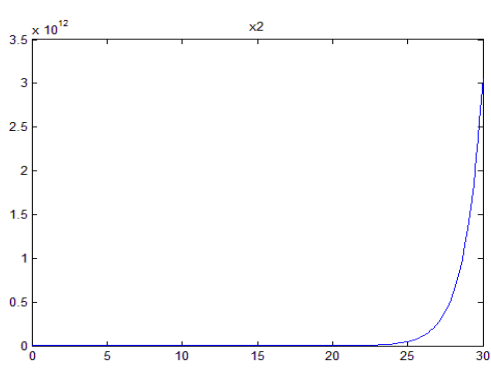

Fig.4. variations of ${ }^{x_{2}}$ related to $\mathrm{t}$ 


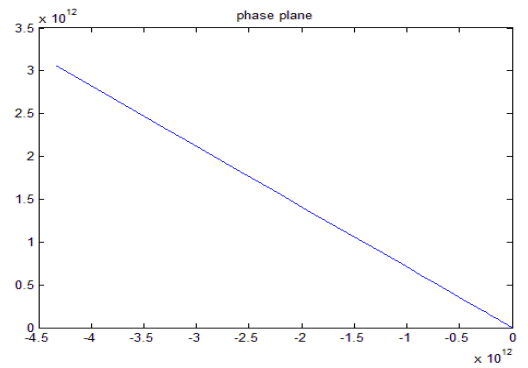

Fig.5. variations of $x_{2}$ related to $x_{1}$

\subsection{Defining the System Parameters}

In this part, the system parameters for each subspace is defined as:

$$
\bar{B}_{1}=\bar{B}_{2}=\bar{B}_{3}=\left[\begin{array}{c}
1 \\
-1 \\
0
\end{array}\right]
$$

$$
\begin{aligned}
& \bar{A}_{1}=\left[\begin{array}{ccc}
-4 & 4 & -1 \\
2 & -6 & 1 \\
0 & 0 & 0
\end{array}\right] \\
& \bar{A}_{2}=\left[\begin{array}{ccc}
-1 & -1 & 0 \\
-1 & -1 & 0 \\
0 & 0 & 0
\end{array}\right] \\
& \bar{A}_{3}=\left[\begin{array}{ccc}
-4 & 4 & 1 \\
2 & -6 & -1 \\
0 & 0 & 0
\end{array}\right] \\
& \bar{F}_{12}=\left[\begin{array}{cc}
1 & 0 \\
3 / 5 & 1 / 5 \\
0 & 1
\end{array}\right], \bar{F}_{23}=\left[\begin{array}{cc}
1 / 5 & -1 / 5 \\
0 & 1
\end{array}\right]
\end{aligned}
$$

When the spaces are hyper-plane we have:

$$
\begin{aligned}
& E_{1}=\left[\begin{array}{llr}
-3 & 5 & -1
\end{array}\right] \\
& E_{2}=\left[\begin{array}{ccr}
-3 & 5 & 1 \\
3 & -5 & 1
\end{array}\right] \\
& E_{3}=\left[\begin{array}{llr}
3 & -5 & -1
\end{array}\right]
\end{aligned}
$$

With following normal vectors

$$
\begin{aligned}
& C_{12}=\left[\begin{array}{lll}
-3 & 5 & -1
\end{array}\right] \\
& C_{23}=\left[\begin{array}{lll}
-3 & 5 & 1
\end{array}\right]
\end{aligned}
$$

\subsection{Controller Design}

The PWA system (39) is rewritten as:

$\dot{x}_{1}=-4 x_{1}+4 x_{2}-\operatorname{sat}\left(-3 x_{1}+5 x_{2}\right)+u$

$\dot{x}_{2}=2 x_{1}-6 x_{2}+\operatorname{sat}\left(-3 x_{1}+5 x_{2}\right)-u$

To find the stabilizing controller considering to theorem 3 , the proposed controller should satisfiy the linear and nonlinear conditions 30 to 35 . In this paper we propose a simple tool to solve such a problem by converting the system to a multi objective nonlinear optimization problem which can be easily solved by a simple optimization algorithm like GA. Where the proposed cost function has the following structure:

$\min f=a_{i}$ heaviside

$$
\begin{aligned}
& {\left[\left(L_{1}-P_{1}\right)+\left(L_{2}-P_{2}\right)+\left(L_{3}-P_{3}\right)+\left(\begin{array}{l}
\left(A_{1}+B K_{1}\right)^{T} P_{1}+ \\
P_{1}\left(A_{1}+B K_{1}\right)+M_{1}
\end{array}\right)+\right.} \\
& \left(\left(A_{2}+B K_{2}\right)^{T} P_{2}+P_{2}\left(A_{2}+B K_{2}\right)+M_{2}\right)+ \\
& \left.\left(\left(A_{3}+B K_{3}\right)^{T} P_{3}+P_{3}\left(A_{3}+B K_{3}\right)+M_{3}\right)\right]+ \\
& b_{i} \mid\left(F_{12}{ }^{T}\left(P_{1}-P_{2}\right) F_{12}-F_{12}{ }^{T}\left(H_{12}+H_{12}{ }^{T}\right) F_{12}\right)+ \\
& \left(F_{23}{ }^{T}\left(P_{2}-P_{3}\right) F_{23}-F_{23}{ }^{T}\left(H_{23}+H_{23}{ }^{T}\right) F_{23}\right) \\
& +\left(\left(A_{1}+B K_{1}\right) F_{12}-\left(A_{2}+B K_{2}\right) F_{12}\right)+ \\
& \left(\left(A_{2}+B K_{2}\right) F_{23}-\left(A_{3}+B K_{3}\right) F_{23}\right)
\end{aligned}
$$

Where the GA parameters are defined in table 1.

Table1. GA parameters

\begin{tabular}{|c|c|c|}
\hline Crossover & Populationsize & Generation \\
\hline 0.8 & 500 & 100 \\
\hline
\end{tabular}

And the controlling game for each subspace is proposed as:

$$
\begin{aligned}
& K_{1}=\left[\begin{array}{lll}
-0.7063 & 0.3362 & 1.4319
\end{array}\right] \\
& K_{2}=\left[\begin{array}{lll}
1.2260 & 0.5675 & -1.1019
\end{array}\right] \\
& K_{3}=\left[\begin{array}{lll}
0.6556 & 0.9828 & -0.1632
\end{array}\right]
\end{aligned}
$$

As it is shown in figures 6 to 8 , the proposed controller stabilizes system (39).

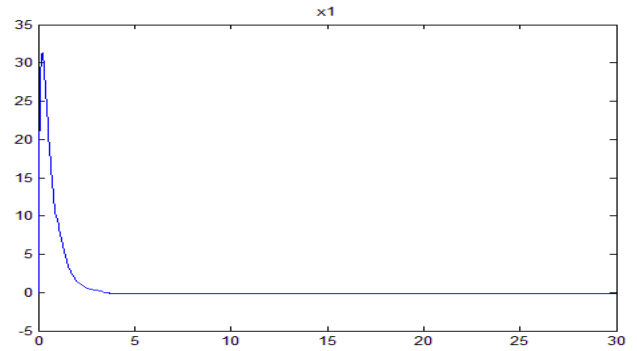

Fig.6. Variations of ${ }^{x_{1}}$ related to $\mathrm{t}$

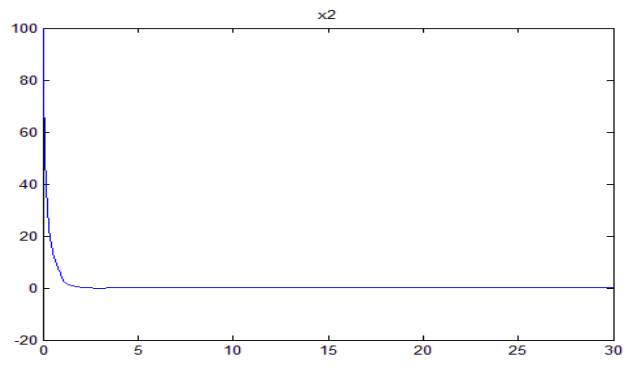

Fig.7. Variations of ${ }^{x_{2}}$ related to $\mathrm{t}$ 


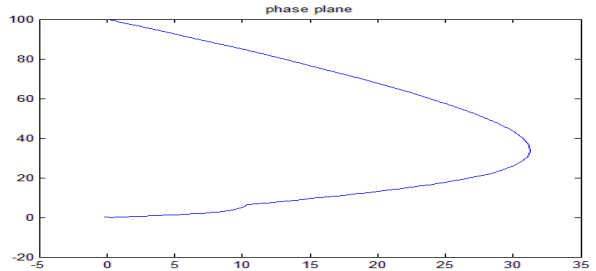

Fig.8. Variations of $x_{2}$ related to $x_{1}$

\section{CONCLUSION}

In this paper, a new method for stabilizing a special class of PWA systems is proposed. The proposed method is based on DPWQ Lyapunov function which decreases the conservation and proposes a wider class of applicable Lyapunov functions as it omits the continuity condition in boundary points compared to CPWQs. The proposed stability conditions form a BMI problem which is converted to a multi objective nonlinear optimization problem that can be solved easily by using optimization methods like GA. Considering the uncertainty of such systems and designing robust controllers is the topic of our future work

\section{REFERENCES}

[1] Hamed Molla Ahmadian, Ali Karimpour, Naser Pariz "Stabilization and Control of Switched Linear Systems with State-Input Logic Constrained: LMI Approach", Journal of Control , Vol. 5, No. 2, Summer 2011.

[2] N. Eghbal, N. Pariz, N. Karimpour, "Discontinuous piecewise quadratic Lyapunov functions for planar piecewise affine systems", Elsevier Journal of Mathematical Analysis and Applications , Volume 399, Issue 2 , Pages 586-593 , 2012.

[3] Najme Eghbal, Modelling class of parametric nonlinear systems as nondefinite section, Ph.D. thesis, Ferdosi University of Mashhad, 110 pages, February 2012.

[4] P.Julian, A. Desages, O. Agamennoni, "High -level canonical piecewise linear representation using a simplicial partition", IEEE Trans. Circuits Syst. I: Fund. Theory Appl. 46 (4) (1999) 463-480.

[5] P.Julian, A.Desages, B.D'Amico, “Orthonormal high level canonical PWL functions with applications to model reduction," IEEE Tran sactions on Circuits and Systems I, vol. 47, no. 5, pp 702-712, 2000.

[6] M.S.Branicky, "Studies in Hybrid Systems : Modeling , Analysis and Control ", Department of Electrical Engineering and Computer Science, Massachusetts Institute of Technology, PHD thesis, June 1995.

[7] J.N. Lin, R. Unbehauen, "Canonical piecewise-linear approximations", IEEE Trans. Circuits Syst. I: Fund. Theory Appl. Vol 39, no 8, pp 697-699, 1992.

[8] M. Storace, O.D. Feo, "PWL approximation of nonlinear dynamical systems, part I: structural stability", J. Phys. Conf. Ser, Vol 22, pp 208-221, 2005.

[9] P.Julian, A.Desages, O.Agamennoni ,“ High-level canonical piecewise linear representation using a simplicial partition", IEEE Trans. Circuits Syst. I:Fund . Theory App 1, vol.46, issue 4, pp 463-480, 1999.

[10] N. Eghbal, N. Pariz, N. Karimpour, "Uniform modeling of parameter dependent nonlinear systems", Journal of Zhejiang University SCIENCE . vol.13, issue11, pp 850858 , nov 2012.

[11] W.P.M.H. Heemels, B.De Schutter, A.Bemporad, "Equivalence of hybrid dynamical models", Automatica , Delft University of Technology, vol 37, no 7, pp 10851091 , july 2001.
[12] A.Bemporad , " Efficient conversion of mixed logical dynamical systems into an equivalent piecewise affine form", IEEE Trans Automat Control , vol 49, no 5, pp $832-838,2004$

[13] M. Johansson, A. Rantzer , "Computation of piecewise quadratic Lyapunov functions for hybrid systems", IEEE Transactions on Automatic Control, vol 43, no 4, pp 555 559, 1998 .

[14] L. Rodrigues,"Dynamic output feedback controller synthesis for piecewise affine systems," Ph.D thesis , Stanford University, Jun 2002.

[15] B. Samadi, "Stability Analysis and Controller Synthesis for a Class of Piecewise Smooth Systems", Ph.D thesis , Concordia University, 2008 .

[16] Boyd s and Ghaoui L.E and Feron E and Balakrishnan V,“ linear matrix inequalities in system and control theory ", Society for Industrial and Applied Mathematics, University City Science Center, Philadelphia , vol 15, 1994

[17] Kai liu , jianghai hu , yu yao , baoqing yang , xin huo, "Stability analysis of discrete-time piecewise-linear systems: a generating function approach", international journal of control ,automation and systems, volume12, issue5, pp 1005-1010, 30Aug 2014.

[18] K.S. Narendra and J. Balakrishnan .A Common Lyapunov Function for Stable Systems with Commuting A Matrices". IEEE Transactions on Automatic Control, vol 39, issue 12, pages 2469-2471, Dec 1994.

[19] Guisheng zhai, ryuuen kou, joe imae, tomoaki kobayashi ,"stability analysis and design for switched descriptor systems", international journal of control , automation and systems, volume7, issue 3, pp 349-355, 30 may 2009.

[20] S.Pettersson and B.Lennartson , "Stability and robustness for hybrid systems", Proceedings of the 35th Conference on Decision and Control, Kobe, Japan , vol 2, pp 1202 $1207,1996$.

[21] Stephen Boyd, E.F,“ History of Linear Matrix Inequalities in Control Theory", in American control conference: Maryland, June 1994.

[22] M. Johansson , "Analysis of piecewise linear systems via convex optimization: a unifying approach," IFAC World Congress, Beijing, China, July 1999.

[23] J. Xu, and L. Xie, "Homogeneous polynomial Lyapunov functions for piecewise affine systems," Proceedings of the IEEE American Control Conference, vol 1, pp 581 - 586 , June 2005

[24] D. Mignone, G. Ferrari-Trecate, and M. Morari. "Stability and Stabilization of Piecewise Affine and Hybrid Systems: An LMI Approach". Technical Report AUT00-12, Automatic Control Laboratory, ETH Zurich, Switzerland, vol 1, pp 504- 509, Dec 2000.

[25] M. Johansson ,“ Piecewise Linear Control " Systems , Springer , Berlin , vol $284,2003$.

[26] Liu Kai, Yao Yu, Sund engfeng, Venkataramanan Balakrishnan,"PWA State Feedback Controller Synthesis for Piecewise Linear Systems", Chinese control conference , pp 1252 - 1257 , July 2011.

[27] Liu Kai,Yao Yu, Sun Dengfeng,Venkataramanan Balakrishnan ,“ Improved State Feedback Controller Synthesis For Piecewise Linear Systems ", International Journal of Innovative Computing , Information and Control, Volume 8 , Number 9 , september 2012

[28] Ya-quan ma , Xin Jin , Ben Niu , yanyan liu , "robust tracking control of switched nonlinear systems via the multiple lyapunov function approach",IEEE Chinese control and Decision conference (CCDC) ,pp 2113-2118 ,May 2013. 